\title{
Search for RPV Squark Production at HERA
}

\author{
Julia Fourletova ${ }^{* \dagger}$ \\ DESY, Germany \\ E-mail: julia@mail.desy.de
}

The ep collider HERA is ideally suited to search for new particles which could be produced resonantly via the fusion of the incoming $27.5 \mathrm{GeV}$ electron and a quark from the incoming $920 \mathrm{GeV}$ proton. Particle masses up to the electron-proton centre-of-mass energy, $\sqrt{s}=318 \mathrm{GeV}$, are kinematically accessible. Two collider experiments, H1 and ZEUS, have searched for the production of squarks, the scalar supersymmetric partners of Standard Model quarks, using $e^{+} p$ and $e^{-} p$ data corresponding to luminosities of $64.3 \mathrm{pb}^{-1}$ and $13.5 \mathrm{pb}^{-1}$, respectively. Recent results on the search for squark production in R-parity violating supersymmetry at HERA are presented.

International Europhysics Conference on High Energy Physics

July 21st - 27th 2005

Lisboa, Portugal

* Speaker.

${ }^{\dagger}$ on behalf of $\mathrm{H} 1$ and ZEUS collaborations 


\section{Phenomenology}

One essential quantum number in SUSY models (MSSM) is the $R$-parity $R_{p}=(-1)^{3 B+L+2 S}$, where $B$ denotes the baryon number, $L$ the lepton number and $S$ the spin of a particle. In the most general supersymmetric theory the $R$-parity is not conserved. Couplings between two ordinary fermions and a squark $(\tilde{q})$ or a slepton $(\tilde{l})$ are then allowed. The $R_{p}^{\prime}$ Yukawa couplings which could allow squark production at HERA are described in the superpotential by the terms $\lambda_{i j k}^{\prime} L_{i} Q_{j} \bar{D}_{k}$, where $i, j$ and $k$ are family indices. $L_{i}, Q_{j}$ and $D_{k}$ are superfields, which contain the left-handed leptons, the left-handed quarks and the right-handed down quark, respectively, together with their SUSY partners $\tilde{l}_{L}^{i}, \tilde{q}_{L}^{j}$ and $\tilde{d}_{R}^{k}$. A non vanishing coupling $\lambda_{1 j 1}^{\prime}\left(\lambda_{11 k}^{\prime}\right)$ would allow the resonant production of a $\tilde{u}_{L}^{j}\left(\tilde{d}_{R}^{k}\right)$ squark at HERA via the fusion of the incoming $e^{+}\left(e^{-}\right)$with a valence quark from the proton [1].

In $R_{p}$ SUSY all supersymmetric particles are unstable. Squarks can decay via their Yukawa coupling $\lambda^{\prime}$ into Standard Model (SM) fermions. The $R_{p}$ squark decays proceed directly via the couplings $\lambda_{11 k}^{\prime}$ and $\lambda_{1 j 1}^{\prime}$. The final state signatures consist of a lepton and a jet and are indistinguishable, event-by-event, from neutral current (NC) and charged current (CC) deep-inelastic scattering (DIS).

Squarks can also decay via their usual $R_{p}$ conserving gauge couplings. The final state of these gauge decays depends on the subsequent gaugino decay. The decay channels are classified by event topology [2]. This classification relies on the number of charged leptons and/or hadronic jets in the final state, and on the presence of missing momentum.

\section{Searches for SUSY Signals}

No significant deviation from the SM expectation is found in any channel using the data collected between 1999 and 2000. The selection channels are combined, separately for the $e^{+} p$ and $e^{-} p$ data sets, to derive constraints on $R_{p}$ SUSY models. In order to investigate the dependence of the sensitivity on the MSSM parameters, a scan of $M_{2}$ and $\mu$ is performed. The parameters $M_{2}$ and $\mu$ are varied in the range $70 \mathrm{GeV}<M_{2}<350 \mathrm{GeV}$ and $-300 \mathrm{GeV}<\mu<300 \mathrm{GeV}$. Parameter sets leading to a scalar LSP or to LSP masses below $30 \mathrm{GeV}$ are not considered. Upper bounds on the couplings $\lambda_{1 j 1}^{\prime}$ and $\lambda_{11 k}^{\prime}$ are obtained for each point in the $\left(\mu, M_{2}\right)$ plane.

As an example, Fig. 1 and Fig. 2 show the upper bounds on the couplings $\lambda_{1 j 1}^{\prime}$ and $\lambda_{11 k}^{\prime}$ resulting from such a scan, for $\tan \beta=6$ and assuming that the slepton mass is $90 \mathrm{GeV}$. Similar results are obtained if the slepton mass is set to that of the squarks. The two full curves indicate the regions excluded in all (upper curve) or part (lower curve) of the parameter space investigated. As can be seen from the narrowness of the region that is excluded in only part of the parameter space, the limits on both $\lambda_{1 j 1}^{\prime}$ and $\lambda_{11 k}^{\prime}$ are widely independent of the SUSY parameters. For a Yukawa coupling of electromagnetic strength, i.e. $\lambda_{1 j 1}^{\prime}\left(\lambda_{11 k}^{\prime}\right)=\sqrt{4 \pi \alpha_{\mathrm{em}}}=0.3, \tilde{u}_{L}, \tilde{c}_{L}$ and $\tilde{t}_{L}\left(\tilde{d}_{R}, \tilde{s}_{R}\right.$ and $\tilde{b}_{R}$ ) squarks with masses below $\sim 275 \mathrm{GeV}(280 \mathrm{GeV})$ are excluded at the $95 \% \mathrm{CL}$. For a coupling strength smaller by a factor of 100 , masses up to $\sim 220 \mathrm{GeV}$ are ruled out. In Figs. 1 and 2 the results for the direct production of squarks are compared with indirect limits from virtual squark exchange in low energy experiments [3]. The HERA results improve the limits on $\lambda^{\prime}$ for squarks of the second and third family (i.e. $\lambda_{121}^{\prime}, \lambda_{131}^{\prime}, \lambda_{112}^{\prime}, \lambda_{113}^{\prime}$ ) for masses up to $\sim 255 \mathrm{GeV}$. 

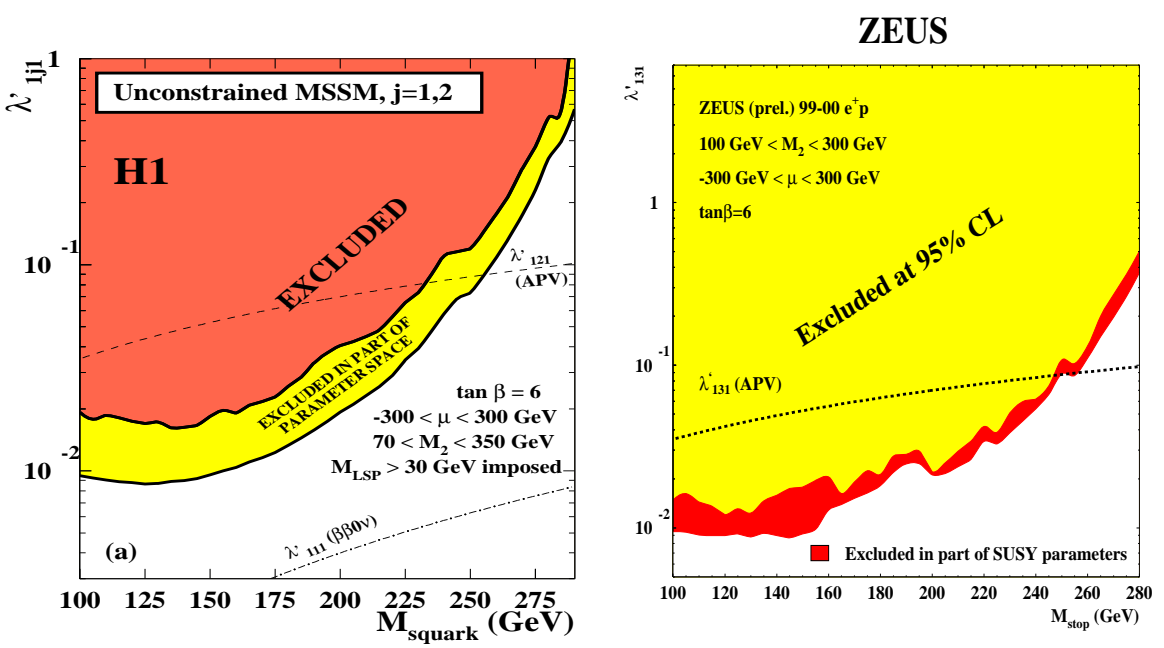

Figure 1: Exclusion limits (95\% CL) on $\lambda_{1 j 1}^{\prime}$ for $j=1,2$ (H1) and $j=3$ (ZEUS) as a function of the squark mass from a scan of the MSSM parameter space as indicated in the Figures.
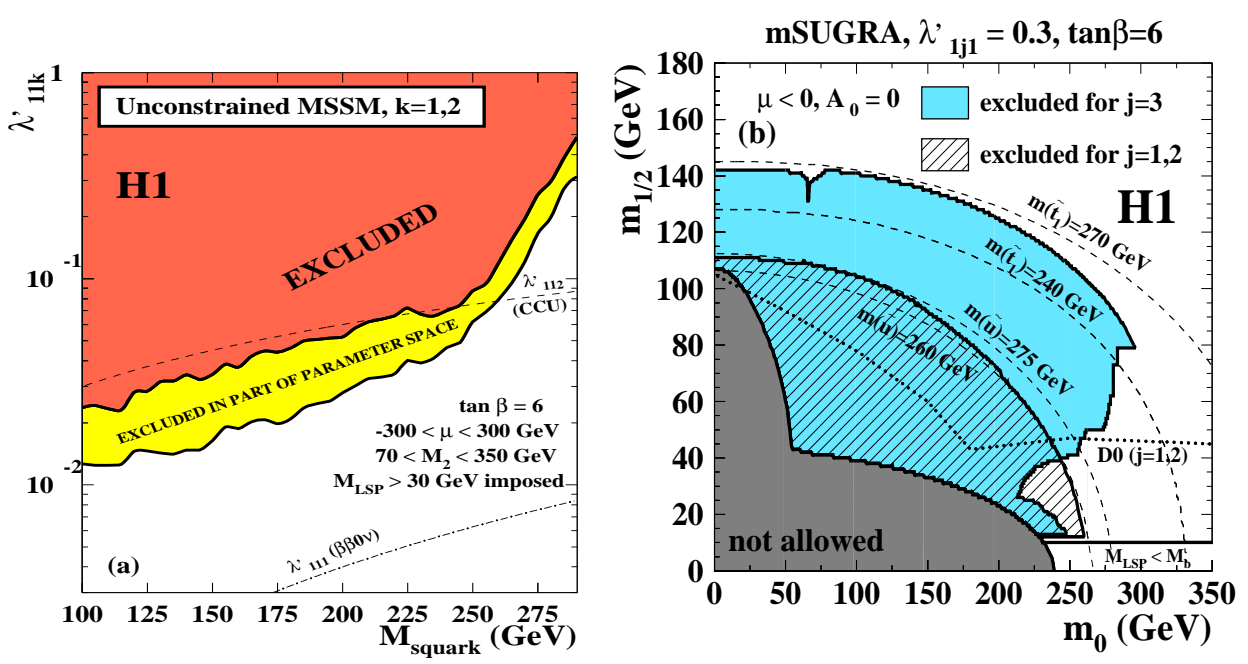

Figure 2: Exclusion limits (95\% CL) on $\lambda_{1 j 1}^{\prime}$ for $k=1,2$ (left plot) as a function of the squark mass from a scan of the MSSM parameter space as indicated in the Figure. Excluded regions (95\% CL) in mSUGRA with $\lambda_{1 j 1}^{\prime}=0.3$ (right plot) for $\tan \beta=6$. The limits from the Tevatron are given by the dotted lines.

In the minimal Supergravity (mSUGRA) model [4] the number of free parameters is reduced by assuming, in addition to the GUT relation between $M_{1}, M_{2}$ and $M_{3}$, a universal mass parameter $m_{0}$ for all scalar fields at the GUT scale. Assuming a fixed value for the $R_{p}^{\prime}$ couplings $\lambda_{1 j 1}^{\prime}$ and $\lambda_{11 k}^{\prime}$, constraints on the mSUGRA parameters can be set, for example on $\left(m_{0}, m_{1 / 2}\right)$, when $\tan \beta, A_{0}$, and the sign of $\mu$ are fixed. For $\mu<0$ and for $\tan \beta=6$, the excluded region (95\% CL) approximately follow curves of constant squark mass as shown in the right plot of Fig. 2. For $\lambda_{1 j 1}^{\prime}\left(\lambda_{11 k}^{\prime}\right)=0.3$, the parameter space defined by $M_{\tilde{q}}<275(285) \mathrm{GeV}$ is nearly fully excluded. Although strongly bounds have been set by LEP on this very constrained model the HERA sensitivity extends, in 
some cases, beyond that of Tevatron [5].

\section{Bosonic Stop decays}

In the searches reported in the previous section, only squark decays into fermions were considered. Bosonic decays might also be relevant in some cases. Of particular interest is the bosonic decay $\tilde{t} \rightarrow \tilde{b} W$, where the sbottom decay into SM particles, $\tilde{b} \stackrel{\lambda^{\prime}}{\rightarrow} \bar{v}_{e} d$, is also R-parity violating and leptonic and hadronic W decays are considered. This stop decay mode is experimentally investigated for the first time. A scenario is investigated, in which the sbottom mass is smaller than the stop mass, $M_{\tilde{b}}<M_{\tilde{t}}$, which are complementary to previous $R_{p}$ SUSY searches for squark production by $\mathrm{H} 1$ [2], and decays of the light squarks into neutralinos and charginos are kinematically suppressed. In order to cover all decay modes, the $R_{p}^{\prime}$ decay $\tilde{t} \stackrel{\lambda^{\prime}}{\rightarrow} e^{+} d$ is also considered.
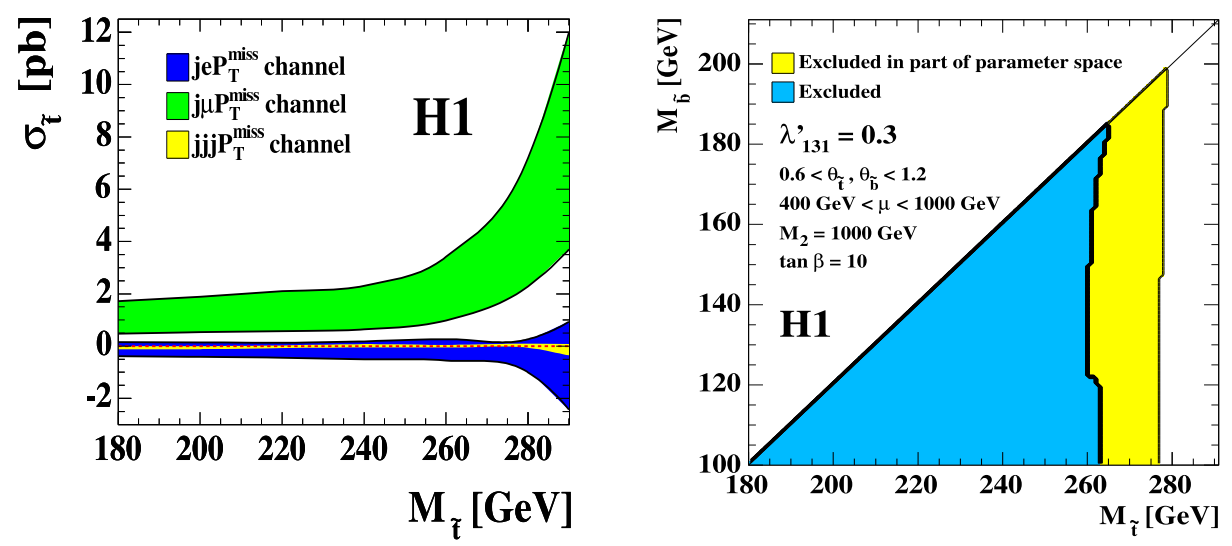

Figure 3: Bands representing the allowed stop cross section regions as a function of the stop mass as obtained in [6] (left plot). Exclusion limits at the $95 \% \mathrm{CL}$ in the $\left(M_{\tilde{t}}, M_{\tilde{b}}\right)$ plane for $\lambda_{131}^{\prime}=0.3$ (right plot). The two full curves indicate the regions excluded in all (dark) or part (light) of the parameter space investigated.

The analysis uses the data collected with the $\mathrm{H} 1$ detector in positron-proton scattering in the years 1994-2000, and corresponds to an integrated luminosity of $\mathscr{L} \sim 106 \mathrm{pb}^{-1}$. A total of 5 events are found in the $j j j P_{\perp}$ final state topology while $6.24 \pm 1.74$ are expected from SM processes. In the $j e P_{\perp}$ channel, 3 events are found while the expectation from the SM is $3.84 \pm 0.92$ events. In the $j \mu P_{\perp}$ channel, 8 events are found while $2.69 \pm 0.47$ are expected. The stop detection efficiency is typically $35 \%-50 \%$. In the $j \mu H_{\perp}$ channel a slight excess of events compared with the SM expectation is observed, as already reported in [6]. All other channels are in good agreement with the SM. The stop interpretation of the excess seen in the $j \mu H_{\perp}$ channel is not supported by the other decay modes as can be seen from the bands of allowed stop cross section regions presented in the left plot of Fig. 3.

The results from the selection channels considered are combined to derive constraints in the MSSM. The parameter $M_{2}$ is set to $1000 \mathrm{GeV}$ and $\mu$ is restricted to the range $400 \mathrm{GeV}<\mu<$ 
$1000 \mathrm{GeV}$. The resulting limits obtained for $\tan \beta=10$ are shown in the right plot of Fig. 3 . Masses $M_{\tilde{t}} \lesssim 275 \mathrm{GeV}$ are excluded for a strength of the Yukawa coupling $\lambda_{131}^{\prime}=\sqrt{4 \pi \alpha_{\mathrm{em}}}=0.3$.

\section{Conclusions}

At HERA a search for R-parity violating squark production is performed. No deviation from SM expectations has been found. New constraints on the squark production have been obtained.

\section{Acknowledgments}

Many thanks to colleagues from H1 and ZEUS group for their help in preparing this talk.

\section{References}

[1] J. Butterworth and H. Dreiner, Nucl. Phys. B397 (1993) 3 and references therein.

[2] C. Adloff et al. [H1 Collaboration], Eur. Phys. J. C 20 (2001) 639 [hep-ex/0102050].

[3] H. Dreiner, published in "Perspectives on Supersymmetry”, Ed. G.L. Kane, World Scientific (1997) 462 [hep-ph/9707435].

M. Hirsch, H. V. Klapdor-Kleingrothaus and S. G. Kovalenko, Phys. Rev. D53 (1996) 1329

[hep-ph/9502385].

P. Langacker, Phys. Lett. B256 (1991) 277.

V. Barger, G. F. Giudice and T. Han, Phys. Rev. D40 (1989) 2987.

[4] H. Baer and X. Tata, Phys. Rev. D47 (1993) 2739;

G. L. Kaneet al. Phys. Rev. D49 (1994) 6173.

[5] B. Abbott et al. [D0 Collaboration], Phys. Rev. Lett. 83 (1999) 4476 [hep-ex/9907019].

[6] V. Andreev et al. [H1 Collaboration], Phys. Lett. B 561 (2003) 241 [hep-ex/0301030]. 